\title{
Influx of uncommon HIV-1 strains from Eastern Europe and identification of a new unique recombinant strain among young Cypriot MSM in Cyprus
}

Ioanna Kousiappa*, Yiota Lazarou, Katerina M Othonos, Johana Hezka, Leondios G Kostrikis

From 17th International Symposium on HIV and Emerging Infectious Diseases (ISHEID) Marseille, France. 23-25 May 2012

\section{Introduction}

The polyphyletic picture of HIV-1 infection in Cyprus is a fact, as new variants and unique recombinant forms were found in the recent past. As part of a growing effort to monitor any changes in the molecular epidemiology of HIV we studied two notable cohorts of the known HIV-1 population diagnosed in 2010 to 2011. Near full-length genome sequencing and phylogenetic analysis was carried out to determine the heterogeneity among HIV-1 strains isolated from patients, 10 originated from eastern European countries (mostly Romania) and 8 young Cypriots $(<25$ yrs $)$, all men who have sex with men (MSM).

\section{Materials and methods}

Sequence of the near full-length genome was amplified by RT-nested PCR from all HIV-1 seropositives and sequenced. Detailed phylogenetic and bootscanning analyses were performed by MEGA v5.0 to determine phylogenetic associations and subtype assignments. To explore putative recombination patterns in the sequences we performed a bootscanning analysis using Simplot, version 3.5.1.

\section{Results}

Phylogenetic analyses of the obtained viral sequences showed genetic diversity. In the eastern European cohort, subtype F1 was the dominant subtype (40\%), followed by subtype C (20\%), A1, A2, CRF02_AG, and CRF03_AB (10\% each). In the young MSM cohort subtype B was the main subtype (50\%), followed by subtype A1 (25\%), CRF01_AE (12.5\%) and one HIV-1 isolate that was not classified in any known subtype or recombinant form $(12.5 \%)$. Complete recombination analysis revealed that this isolate had a new recombinant pattern, comprising segments of subtypes A1 and B, and is distinct from any reported recombinant.

\section{Conclusions}

These findings exhibit an influx of infrequent HIV-1 genetic forms from eastern European countries in Cyprus, and a stable circulation of B and A1 subtype among the young Cypriot MSM cohort. A unique recombination event between $\mathrm{A} 1$ and $\mathrm{B}$ subtypes has occurred and the parental strains seem to be formerly characterized Cypriot MSM patients. For the first time, these data show an impact on the evolutionary progress of HIV-1 epidemic of the island. The significance of this study along with the earlier variable epidemiological status of HIV-1 infection in Cyprus reflects the contribution to HIV classification, and the important implications for HIV-1 disease control and surveillance.

Published: 25 May 2012

\section{doi:10.1186/1742-4690-9-S1-P98}

Cite this article as: Kousiappa et al.: Influx of uncommon HIV-1 strains from Eastern Europe and identification of a new unique recombinant strain among young Cypriot MSM in Cyprus. Retrovirology 2012 9(Suppl 1):P98.

* Correspondence: kousiappa.ioanna@ucy.ac.cy

University of Cyprus, Nicosia, Cyprus 